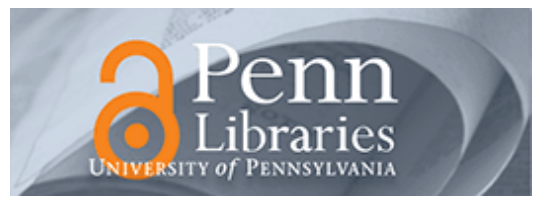

University of Pennsylvania

ScholarlyCommons

Finance Papers

Wharton Faculty Research

$10-2014$

\title{
A Demand Estimation Procedure for Retail Assortment Optimization with Results from Implementations
}

\author{
Marshall Fisher \\ University of Pennsylvania \\ Ramnath Vaidyanathan \\ University of Pennsylvania
}

Follow this and additional works at: https://repository.upenn.edu/fnce_papers

Part of the Business Administration, Management, and Operations Commons, and the Finance and Financial Management Commons

\section{Recommended Citation}

Fisher, M., \& Vaidyanathan, R. (2014). A Demand Estimation Procedure for Retail Assortment Optimization with Results from Implementations. Management Science, 60 (10), 2401-2415. http://dx.doi.org/10.1287/ mnsc.2014.1904

This paper is posted at ScholarlyCommons. https://repository.upenn.edu/fnce_papers/106

For more information, please contact repository@pobox.upenn.edu. 


\title{
A Demand Estimation Procedure for Retail Assortment Optimization with Results from Implementations
}

\begin{abstract}
We consider the problem of choosing, from a set of $N$ potential stock-keeping units (SKUs) in a retail category, KSKUs to be carried at each store to maximize revenue or profit. Assortments can vary by store, subject to a maximum number of different assortments. We view a SKU as a set of attribute levels and also model possible substitutions when a customer's first choice is not in the assortment. We apply maximum likelihood estimation to sales history of the SKUs currently carried by the retailer to estimate the demand for attribute levels and substitution probabilities, and from this, the demand for any potential SKU, including those not currently carried by the retailer. We specify several alternative heuristics for choosing SKUs to be carried in an assortment. We apply this approach to optimize assortments for three real examples: snack cakes, tires, and automotive appearance chemicals. A portion of our recommendations for tires and appearance chemicals were implemented and produced sales increases of $5.8 \%$ and $3.6 \%$, respectively, which are significant improvements relative to typical retailer annual comparable store revenue increases. We also forecast sales shares of 1, 11, and 25 new SKUs for the snack cake, tire, and automotive appearance chemical applications, respectively, with mean absolute percentage errors (MAPEs) of $16.2 \%, 19.1 \%$, and $28.7 \%$, which compares favorably to the $30.7 \%$ MAPE for chain sales of two new SKUs reported by Fader and Hardie (1996).
\end{abstract}

\section{Keywords}

assortment planning, retailer operations, statistics, estimation

\section{Disciplines}

Business Administration, Management, and Operations | Finance and Financial Management 


\title{
A Demand Estimation Procedure for Retail Assortment Optimization with Results from Implementations
}

\author{
Marshall Fisher \\ OPIM Department, The Wharton School, 3730 Walnut Street, Philadelphia, PA 19104, USA \\ fisher@wharton.upenn.edu \\ Ramnath Vaidyanathan \\ Desautels Faculty of Management, 1001 Sherbrooke Street West, Montreal, QC H3A 1G5, Canada \\ ramnath.vaidyanathan@mcgill.ca
}

June 2009, Revised September 2012, May 2013

\begin{abstract}
We consider the problem of choosing, from a set of $N$ potential SKUs in a retail category, K SKUs to be carried at each store so as to maximize revenue or profit. Assortments can vary by store, subject to a maximum number of different assortments. We introduce a model of substitution behavior, in case a customer's first choice is unavailable and consider the impact of substitution in choosing assortments for the retail chain. We view a SKU as a set of attribute levels, apply maximum likelihood estimation to sales history of the SKUs currently carried by the retailer to estimate the demand for attribute levels and substitution probabilities, and from this, the demand for any potential SKU, including those not currently carried by the retailer. We specify several alternative heuristics for choosing SKUs to be carried in an assortment. We apply this approach to optimize assortments for three real examples: snack cakes, tires and automotive appearance chemicals. A portion of our recommendations for tires and appearance chemicals were implemented and produced sales increases of $5.8 \%$ and $3.6 \%$ respectively, which are significant improvements relative to typical retailer annual comparable store revenue increases. We also forecast sales shares of 1, 11 and 25 new SKUs, for the snack cakes, tires and automotive appearance chemicals applications, respectively, with MAPEs of 16.2\%, 19.1\% and 28.7\%, which compares favorably to the 30.7\% MAPE for chain sales of two new SKUs reported by Fader and Hardie (1996).
\end{abstract}

\section{Introduction}

A retailer's assortment is the set of products they carry in each store at each point in time. Retailers periodically revise the assortment for each category, deleting some products and adding others to take account of changes in customer demand over time as well as new products introduced by suppliers. This periodic assortment reset seeks to choose a set of SKUs to carry in the new assortment to maximize revenue or profit over a future planning horizon, subject to a shelf space constraint, which can often be expressed as an upper bound on the number of SKUs carried. 
If a customer's most preferred product is not in a retailer's assortment, they may elect to buy nothing or to purchase another product sufficiently similar to their most preferred product that they are willing to buy it. This possibility of substitution must be taken into account in both assortment optimization and in estimation. In estimation, substitution probabilities need to be estimated and the sales of a SKU to customers who most preferred that SKU must be distinguished from sales to customers who preferred a different SKU but substituted when they did not find their preferred SKU in the assortment.

Most retailers use the same assortment in all stores, except for eliminating some SKUs in smaller stores. Recently however, localizing assortments by store or store cluster has become a high priority for many retailers. For example, Zimmerman (September 7, 2006), O'Connell (April 21, 2008), McGregor (May 15, 2008) and Zimmerman (October 7, 2008) describe recent efforts by Wal-Mart, Macy's, Best Buy and Home Depot to vary the assortment they carry at each store to account for local tastes. Despite the flurry of interest in localization reported in the business press and which we have encountered in our interaction with retailers, there have been no studies to document the level of benefits from localization or to provide tools to help a retailer determine the right degree of localization.

The assortment planning process varies across retail products, which are commonly segmented into apparel, grocery, and everything else, usually called hard goods. ${ }^{1}$ An analytic approach to apparel assortments is challenging because rapidly changing tastes make sales history of limited value. Grocery assortment planning (called category management) is the most analytic, due in part to companies like Nielsen and IRI who enlist households to record over time their grocery purchases in all stores. Much academic research on grocery consumer behavior has relied heavily on this data. Among other things, the data allows one to model substitution behavior by observing what a customer buys, if anything, when a product they purchase every week is unavailable due to a stockout. Many hard goods retailers conduct an annual review of their various categories, reallocating store space by category and deciding which SKUs to delete and add to each assortment.

SKU deletion decisions are relatively easy, since sales data is available to indicate the popularity of existing SKUs, but retailers lack (with the partial exception of grocery), analytic tools to forecast the sales of SKUs that might be added to the assortment, to estimate the rate of customer substitution, and to intelligently localize assortments by store. The goal of this paper is to provide these tools.

Our approach follows the marketing literature in viewing a SKU as defined by a set of attribute levels and assuming that a given customer has a preferred set of attribute levels. We use prior sales to estimate the market share in each store of each attribute level and forecast the demand share for a SKU as the product of the demand shares for the attribute levels of that SKU. We assume that if a customer does not find their ideal product in the assortment, they buy the product in the assortment closest to their ideal with some probability and we also estimate these substitution probabilities. We apply various heuristics to these demand and substitution estimates to determine optimized assortments.

In the extreme, a retailer might carry a unique assortment in each store, but most retailers claim that this is administratively too complicated. In particular, retailers develop a diagram called a planogram showing how all products should be displayed in a store, a process that is labor intensive. A planogram would need to be developed for each assortment, which means the administrative cost of each assortment

\footnotetext{
${ }^{1}$ This discussion is based on Fisher and Raman (2010) and conversations with several retail executives including Paul Beswick, Partner and Head, Oliver Wyman North American Retail Practice, Robert DiRomualdo, former CEO, Borders Group, Kevin Freeland, COO, Advance Auto, Matthew Hamory, Principal, Oliver Wyman North American Retail Practice, Herbert Kleinberger, Principal, ARC Consulting, Chris Morrison, Senior VP of Sales, Americas, Tradestone, Robert Price, Chief Marketing Officer, CVS, and Cheryl Sullivan, Vice President of Product Management, Revionics, Inc.
} 
is high. Our process can control the degree of localization by limiting the number of different assortments to be any level between a single assortment for the chain to a unique assortment for each store.

We applied this approach to the snack cakes category at a regional convenience store chain, the tire assortment at a national tire retailer and the appearance chemicals category of a major auto aftermarket parts retailer. The tire and auto parts retailers implemented portions of our recommended assortment changes and obtained revenue increases of $5.8 \%$ and $3.6 \%$ respectively, significant improvements given traditional comparable store annual increases in these segments.

Our approach best fits situations where demand is changing over time, but gradually enough that history is useful, where the individual consumer data common in grocery is not available, and where some products are better substitutes for a given product than others. Also, we do not consider inventory decisions, so our approach fits when inventory decisions are unrelated to assortment decisions, because the retailer carries a fixed, often small amount of inventory for each SKU. This was the case for all of our applications, as well as other categories like jewelry, books and CDs.

Despite the enormous economic importance of assortment planning, we are aware of only two papers, Chong et al. (2001) and Kök and Fisher (2007), that formulate a decision support model for assortment planning, describe a methodology for estimating parameters and optimizing assortments and test the process on real data. While these papers are an excellent start, there is obviously a need for much more research on the vast topic of assortment planning. This paper extends these papers, and hence the exisiting literature, in four ways.

1. We provide a thorough treatment of the important emerging topic of assortment localization. We allow a constraint on the number of different assortments so as to bound the administrative cost of localization and measure how the amount of localization impacts revenue. We compare and analyze the causes of differing levels of localization benefits across our three applications. Chong et al. (2001) do not deal with localization. Kök and Fisher (2007) allow a unique assortment for each store but do not provide a constraint on the number of assortments or assess how localization impacts revenue.

2. We forecast the demand for new SKUs that have not been carried before in any store, based on past sales of products currently carried. Chong et al. (2001) do not explicitly forecast new SKUs, although it would appear from their process that they could derive forecasts for new SKUs. However, they need consumer-level transaction data over multiple shopping trips and this detailed data is not available in most non-grocery applications. Kök and Fisher (2007) forecast how a SKU carried in some stores will sell in other stores in which it is not carried, but do not provide a way to forecast demand for a completely new SKU carried in no stores.

3. We introduce a new demand model not previously considered in assortment decision support research. Both Chong et al. (2001) and Kök and Fisher (2007) use the multinomial logit demand model, which implicitly assumes that substitution demand is divided over available products in proportion to those products' market share. This assumption fits a situation in which products are similar to each other, but vary in a taste parameter, such as different flavors of yogurt or colors of apparel. By contrast, our demand model fits a situation in which some products are better substitutes for a given product than others. This was a very real feature of our applications; for example, the natural substitutes for a 14 inch tire are other 14 inch tires, not 15 inch tires. As an- 
other example, in our snack cakes application we found the probability of substituting from Brand 1 to Brand 2 was $89 \%$, but only $22 \%$ of substituting from Brand 2 to Brand 1 . Hence Brand 2 customers were much more loyal. The multinomial logit approach better fits some real assortment problems and our approach fits others better, so both are needed.

4. Two of the retailers in our study implemented a portion of our recommended assortment changes and we estimate that the revenue increase in revenue from these changes is larger than the annual same store revenue increases typically seen in these industries. We believe this is the first implementation validation of an analytic approach to assortment planning.

\section{Literature Review}

We review here the prior research on consumer choice models, demand estimation and assortment optimization that is most related to this paper. See Kök et al. (2008) for a more extensive review.

Consumer choice models constitute the fundamental platform for assortment planning, and may be classified as (1) utility based models, and (2) exogenous demand models. Utility based models assume that every customer associates a utility $U_{i}$ with each product $i \in N$. In addition, there is a no-purchase option denoted $i=0$, with associated utility $U_{0}$. When offered an assortment $A$, every customer chooses the option giving him the highest utility in $A \cup\{0\}$. The market share for each SKU $i \in A$ can then be evaluated once we know the distribution of utilities across the consumer population.

The Multinomial Logit (MNL) model (Guadagni and Little 1983) assumes that the utilities $U_{i}$ can be decomposed into a deterministic component $u_{i}$ that represents the average utility derived by the population of customers, and a random component $\xi_{i}$ that represents idiosyncrasies across customers. The $\xi_{i}$ are assumed to be identical and independent Gumbel random variables with mean zero and scale parameter $\mu$. Under these assumptions, the market share for each SKU $i \in A$ can be written as $\frac{\exp \left(\frac{u_{i}}{\mu}\right)}{\sum_{i \in A} \exp \left(\frac{u_{i}}{\mu}\right)}$.

In the Locational Choice model (Hotelling 1929, Lancaster 1966, 1975), every SKU $i \in N$ is represented as a bundles of attribute levels $z_{i}=\left(z_{i}^{1}, z_{i}^{2}, \ldots, z_{i}^{T}\right) \in \mathbb{R}^{T}$. Each consumer has an ideal point $y \in$ $\mathbb{R}^{T}$ that defines his most preferred attribute levels. The utility this consumer associates to SKU $i$ is $U_{i}^{y}=c-\tau\left\|y-z_{i}\right\|$, where $c$ is the utility derived from his most preferred product $y$, and $\tau$ is the disutility associated with each unit of deviation from $y$. A consumer not finding his ideal product $y$ in the assortment $A$ substitutes the variant $j \in A$ that is located closest to his ideal point in the attribute space if $U_{j}^{y}>0$, or declines to purchase if $U_{j}^{y} \leq 0$.

In the exogenous demand model, every consumer is assumed to have a favorite product $i$, and $f_{i}$ is the share of consumers whose favorite product is $i$. A consumer whose favorite product is $i$ buys it if $i \in A$; if $i \notin A$ they substitute to SKU $j \in A$ with probability $\alpha_{i j}$. Under these assumptions, the market share of each SKU $i \in A$ is given by $q_{i}(A)=f_{i}+\sum_{j \notin A} f_{j} \alpha_{j i}$.

All three demand models assume that customers have a favorite product and they buy that product if it is in the assortment. They also all assume that if a customer's favorite product is not in the assortment, they may substitute a different product. Where the models differ is in their assumptions about substitution behavior. The exogenous demand model is the most flexible model, allowing for any substitution structure, but it has many parameters and is hence difficult to estimate in practice. The 
MNL model assumes that the demand for a missing product which is not lost transfers to other products in the assortment in proportion to their popularity. By contrast, the Locational Choice model assumes that a given product may be more like some products than others and that substitution demand transfers to the product in the assortment that is most similar to a customer's preferred product.

Demand estimation has been studied by Fader and Hardie (1996), who use maximum likelihood to estimate the parameters of an MNL model from sales transaction data under the assumption that product utility is the sum of utilities of the product attributes. Anupindi et al. (1998), Chong et al. (2001) Bell et al. (2005), Kök and Fisher (2007) and Vulcano et al. (2009) estimate demand as part of an assortment optimization process, and are discussed below.

Assortment optimization research has been based on both stylized models intended to provide insight into structural properties of optimal assortments and decision support models intended to guide a manager planning retail assortments.

Stylized model research began with van Ryzin and Mahajan (1999), who show that an optimal assortment under a MNL consumer choice model consists of a certain number of the highest utility products. Mahajan and van Ryzin (2001), Cachon et al. (2005), Caro and Gallien (2007) and Maddah and Bish (2007) extend van Ryzin and Mahajan (1999) in various ways.

Gaur and Honhon (2006) show that for a locational choice model, the products in the optimal assortment are located far from each other in the attribute space, indicating maximum differentiation, with no substitution between products in the assortment. This implies that the most popular product may not be carried in the optimal assortment, contrasting the results of van Ryzin and Mahajan (1999).

Decision support research began with Green and Krieger (1985), who formulate the problem of which out of a set of potential products, a firm should select so as to maximize (1) consumer welfare or (2) firm profits, and propose solution heuristics.Green and Krieger (1987a,b, 1992), McBride and Zufryden (1988), Dobson and Kalish (1988) and Kohli and Sukumar (1990) extend this line of research. Belloni et al. (2008) compare the performance of different heuristics for product line design and find that the greedy and the greedy-interchange heuristics perform extremely well.

Smith and Agrawal (2000) use an exogenous demand model and an integer programming formulation of assortment planning. They solve a number of small problems by complete enumeration to demonstrate how assortment and stocking decisions depend on the nature of assumed substitution behavior, and also propose a heuristic to solve larger problems.

Chong et al. (2001) develop an assortment modeling framework based on the Guadagni and Little (1983) brand-share model. They use consumer-level transaction data over multiple grocery shopping trips to estimate the parameters of the model and use a local improvement heuristic to suggest an alternative assortment with higher revenue. Although their model can implicitly predict SKU level demand, their explicit focus is on brand-shares, and hence they do not create or measure the accuracy of SKU level demand forecasts. They report an average $50 \%$ mean squared error of predicting brand choice at the customer level across different product categories.

Kök and Fisher (2007) use an exogenous demand model to study a joint assortment selection and inventory planning problem in the presence of shelf-space constraints. They note that the constrained shelf space implies that average inventory per SKU is inversely related to the breadth of the assortment and assess the tradeoff between assortment breadth vs in-stock level for the SKUs carried. They consider substitution and assume that substitution demand accrues to available products in proportion to 
their original market share, as in the MNL model. They provide a process for estimating demand and substitution rates and apply their method to data from a large Dutch grocery retailer.

\section{Problem Formulation and Demand Model}

We seek optimal assortments for a retail category over a specified future planning horizon. We assume that the goal is to maximize revenue, since this was the concern in our three applications. It is straightforward to adapt our approach to maximizing other functions, such as unit sales or gross profit. We define the following parameters.

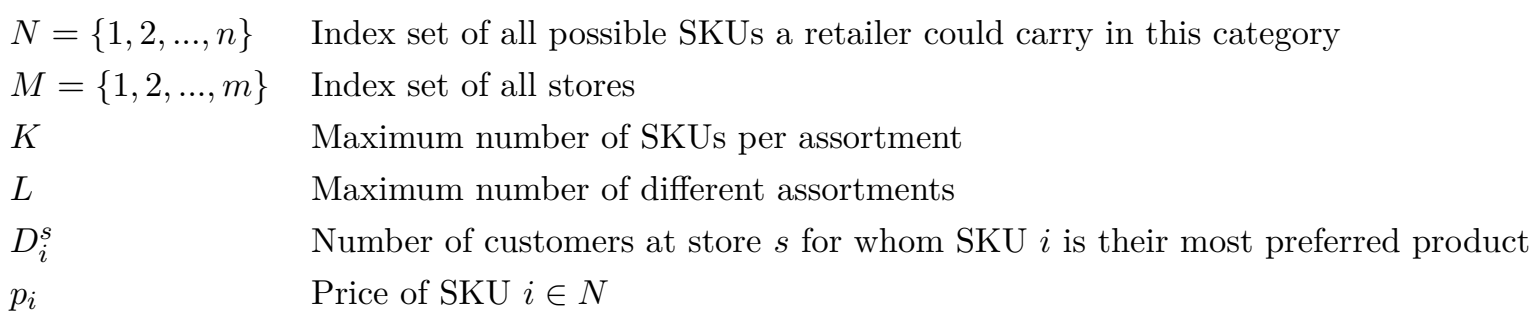

The parameters $K$ and $L$ would be specified by the retailer. For expositional simplicity, $K$ does not vary by store, but it would be easy to modify our process to enable $K$ varying by store, and in fact we do this in our computational work. $L$ would be chosen to lie between 1 and $m$ to tradeoff the greater revenue that comes with larger $L$ against the administrative simplicity that comes with smaller $L$. As will be seen, our solution approach makes it easy to solve this problem for all possible values of $K$ and $L$, thus providing the retailer with rich sensitivity analysis to guide their choice of these parameters.

We define an assortment to be a set $S \subseteq N$ with $|S| \leq K$ and let $l_{s}$ denote the index of the assortment assigned to store $s$. A solution to the assortment optimization problem is defined by a portfolio of assortments $S_{l}, l=1,2, \ldots, L$ and $l_{s} \in\{1,2, \ldots, L\}$, for all $s \in M$.

Our demand model assumes that a consumer shopping in this category in store $s$ has a most preferred SKU $i \in N$, but might be willing to substitute to other SKUs if $i \notin S_{l_{s}}$. We view a SKU as a collection of attribute levels, use historical sales data to estimate the demand share of each attribute level, and finally estimate the demand share of any SKU as the product of the demand shares of its attribute levels. We introduce some notation to formalize this approach.

A Number of attributes

a Attribute type index

$N_{a} \quad$ Number of levels of attribute $a, a=1,2, \ldots, A$

$f_{a u}^{s} \quad$ Fraction of customers at store $s$ who prefer level $u$ of the attribute $a, u=1,2, \ldots, N_{a}, a=1,2, \ldots, \mathrm{A}$

$\pi_{\text {auv }}^{s} \quad$ Probability that a customer at store $s$ whose first choice on attribute $a$ is $u$ is willing to substitute to $v$, defined for all $a, u$, and $v$.

By definition, $\pi_{a v v}^{s}=1$. Moreover, $\pi_{a u v}^{s}$ can be 0 if attribute level $v$ is not a feasible substitute for $u$. For example, size is an attribute of a tire and a 14 inch tire is not a feasible substitute for a customer with a 15 inch wheel.

The fraction of customers who most prefer SKU $i$ with attribute levels $i_{1}, i_{2}, \ldots, i_{A}$ is defined to be $f_{i}^{s}=\prod_{a=1}^{a=A} f_{a i_{a}}^{s}$. If a customer's most preferred SKU $i$ with attributes $i_{1}, i_{2}, \ldots i_{A}$ is not in the assortment, 
they are willing to substitute to SKU $j$ with attributes $j_{1}, j_{2}, \ldots j_{A}$ with probability $\pi_{i j}^{s}=\Pi_{a=1}^{a=A} \pi_{a i_{a} j_{a}}^{s}$. If a customer with most preferred SKU $i$ finds $i \in S_{a(s)}$ when they shop the store, then we assume they buy it. Otherwise, they buy the best substitute for $i$ in $S$, defined to be $j(i, S)=\arg \max _{j \in S} \prod_{a=1}^{A} \pi_{a i_{a} j_{a}}^{s}$ with probability $\pi_{i j(i, S)}^{s}$.

In using store sales data to estimate the parameters of our model, we will estimate $D^{s}$, the total category unit demand in store $s$, if all possible SKUs were offered, and then set $D_{i}^{s}=f_{i}^{s} D^{s}$.

The revenue earned by store $s$ using assortment $S_{l_{s}}$ can then be written as

$$
R_{s}\left(S_{l_{s}}\right)=\left(\sum_{i \in S_{l_{s}}} p_{i} D_{i}^{s}+\sum_{i \notin S_{l_{s}}} D_{i}^{s} \pi_{i j\left(i, S_{l_{s}}\right.}^{s} p_{j\left(i, S_{l_{s}}\right)}\right)
$$

The first term in this expression is the revenue from customers whose most preferred SKU is contained in the assortment and the second term is the expected substitution revenue from customers whose most preferred SKU was not in the assortment.

The assortment optimization problem is to choose $S_{l},\left|S_{l}\right| \leq K, l=1,2, \ldots, L$ and $l_{s}$ for all $s \in M$ to maximize $\sum_{s \in M} R_{s}\left(S_{l_{s}}\right)$.

We allow substitution probabilities to vary by store because we found in our applications that they did in fact vary by store. For example, in the tire application, the willingness of a consumer to substitute to a higher priced product varies by store and is correlated with median income in the zip code in which the store is located.

Our demand model implies that a consumer's preferences for the various attributes are independent, which may not be true. For example a college student shopping for twin size bed-sheets might have a different color preference than a suburban homemaker shopping for queen size sheets, so the color and size attributes for sheets would interact.

Our defense of this assumption is three-fold: (1) all prior publications we are aware of that use attributes in demand estimation make a similar assumption. Fader and Hardie (1996) is typical of the approach followed in the literature. They assume that the utility for a SKU is a linear function of its attributes and then use this utility in a Multinomial Logit model to determine SKU demand shares. They state that "In both the marketing and economics literature, it is common to assume an additive utility function" and note that this implies no interaction between attributes. (2) in our applications, we check the accuracy of this approximation by comparing demand estimates with actual sales for the SKUs currently carried and find that forecasts based on this model are accurate compared to previously published research. (3) if there is significant interaction between attributes, we demonstrate ways to modify our demand model to take this into account. In the snack cakes application, the attributes are flavor, package size (single serve or family size) and brand. Package size and brand interact since one brand is stronger in single serve and another in family size. We deal with this by combining brand and size into a new attribute brand-size. In the tire application, the attributes are size, brand (4 brands) and mileage warranty (low, medium, high). Brand and mileage warranty interact because a given brand does not offer all warranty levels, and so we combine brand and warranty level to create the attribute brand-warranty.

In the tire application, there is also an interaction between size and brand-warranty. A tire with a given size attribute level fits a defined set of car models of a certain age and value. The six brand-warranty levels, corresponding to different price points and quality, might interact with the age and value of the 
cars a size tire fits. We show how to deal with this by partitioning the sizes into a finite number of homogenous segments (which are latent) and allowing the brand-warranty shares to be conditional on segment membership (Fader and Hardie 1996, Kamakura and Russell 1989)

\section{Analysis}

We describe our methods for estimating model parameters and choosing assortments.

\subsection{Estimating demand and substitution probabilities}

We use Maximum Likelihood Estimation to estimate demand and substitution probabilities. Our primary input for estimation is store-SKU sales of products currently carried by the retailer during a prior history period. Parameters are estimated at the store level, but for expositional simplicity we will drop the store superscript in the discussion that follows. We describe here a generic, broadly applicable approach; in Section 5, we will exploit special structure of the applications to refine this approach. Let $S$ denote the assortment carried in a particular store and $x_{i}$ the sales of SKU $i \in S$, during a history period.

We can write the probability $F_{j}(S)$ that a customer purchases $j \in S$, as $F_{j}(S)=f_{j}+\sum_{i \notin S, j=j(i, S)} f_{i} \pi_{i j}$. Let $F(S)=\sum_{j \in S} F_{j}(S)$ denote the probability that a customer shopping in this category makes any purchase from assortment $S$. Then, assuming that each consumer purchase is an independent random draw, the likelihood of observing sales data $x=\left\{x_{i}\right\}_{i \in S}$ is given by $L H(f, \pi)=C \prod_{j \in S}\left[\frac{F_{j}(S)}{F(S)}\right]^{x_{j}}$ where the proportionality constant is $C=\frac{\left(\sum x_{j \in S}\right) !}{\prod_{j \in S} x_{j} !}$. The maximum likelihood estimates (MLE) for the parameters $(f, \pi)$ can be obtained by maximizing the log-likelihood function

$$
L L H(f, \pi)=\sum_{j \in S} x_{j} \log F_{j}(S)-\left(\sum_{j \in S} x_{j}\right) \log F(S)
$$

subject to the constraints

$$
\begin{aligned}
\sum_{u=1}^{N_{a}} f_{a u} & =1, \quad a=1,2, \ldots, A \\
f_{i} & =\Pi_{a=1}^{A} f_{a i_{a}} \quad i=1,2, \ldots, n \\
\pi_{i j} & =\Pi_{a=1}^{A} \pi_{a i_{a} j_{a}} \quad i=1,2, \ldots, n \text { and } j=1,2, \ldots, n \\
f_{a u}, \pi_{a u v} & \in[0,1] \forall a, u, \text { and } v
\end{aligned}
$$

Given the complex nature of the log-likelihood function, it is not possible to derive analytical results. Hence we resort to numerical optimization methods based on gradients after transforming the problem into an unconstrained optimization problem by reparametrizing $f_{a u}$ and $\pi_{a u v}$ in terms of $\hat{f}_{a u}$ and $\hat{\pi}_{a u v}$, 
where

$$
\begin{aligned}
f_{a u} & =\frac{\exp \left(\widehat{f}_{a u}\right)}{\sum_{u=1}^{N_{a}} \exp \left(\widehat{f}_{a u}\right)}, u=1,2, \ldots, N_{a}-1 \text { and } \widehat{f}_{a N_{a}}=1 \\
\pi_{\text {auv }} & =\frac{\exp \left(\widehat{\pi}_{\text {auv }}\right)}{1+\exp \left(\widehat{\pi}_{\text {auv }}\right)}, \quad \forall a, u, \text { and } v
\end{aligned}
$$

We found examples showing that the log-likelihood function may not be concave, which implies that numerical optimization methods may not converge to a global maximum. We handle this issue by running the optimization algorithm from several randomly generated starting points. This does not guarantee global convergence, but lowers the chances of the algorithm getting stuck at a local maximum. Mahajan and van Ryzin (2001) use a similar approach to compute the optimal inventory levels using the sample path gradient algorithm.

Once we obtain MLE estimates for demand shares and substitution probabilities, as noted in the previous section, we estimate total demand for the product category as $D=\frac{\sum_{i \in S} x_{i}}{F(S)}$, and $D_{i}$ as $f_{i} D$.

\subsection{Estimating Prices for New SKUs}

We endeavored to set prices on SKUs not currently carried by the retailer in a way that would be consistent with their current pricing policy. We assume that prices on existing SKUs were set in relationship to the value of the SKU to a consumer and that consumer value is related to attribute levels. Hence, we regressed the log of price on attribute levels to obtain the pricing equation:

$$
\log \left(p_{i}\right)=\alpha_{0}+\sum_{a=1}^{A} \sum_{u=1}^{N_{a}-1} \beta_{a u} z_{i a u}, \quad i=1,2, \ldots, n
$$

where $z_{i a u}$ is a dummy variable taking the value one if SKU $i$ has level $u$ of attribute $a$, and zero otherwise. $^{2}$

This is a hedonic pricing equation, and has been extensively used in economics (Rosen 1974, Goodman 1998, Pakes 2003).

\subsection{Heuristics for Choosing Assortments}

Absent substitution, the assortment problem could be optimally solved by a greedy algorithm that chooses SKU's in decreasing order of their revenue contribution. But substitution makes the objective function nonlinear, because the contribution of a SKU depends in part on its substitution demand, which depends on which other SKUs are in the assortment. As a result, the assortment problem is complex to solve optimally. Hence, we define greedy and interchange heuristics for choosing the assortments $S_{l}, l=1,2, \ldots, L$ and the specification $l_{s}, s \in M$ of the assortment assigned to store $s$.

For assortment planning, Kök and Fisher (2007) use a greedy heuristic and Chong et al. (2001) an interchange heuristic. For the product line design problem, Green and Krieger (1985) use greedy and interchange. Belloni et al. (2008) find that for the product line design problem, greedy and interchange

\footnotetext{
${ }^{2}$ The summation is only over $N_{a}-1$ levels of attribute $a$, since the reference levels for all attributes are accounted for by the intercept term $\alpha_{0}$.
} 
together find $98.5 \%$ of optimal profits on average for randomly generated problems, and $99.9 \%$ for real problems.

The greedy heuristic for finding a single assortment for a store or set of stores adds SKUs in decreasing order of revenue contribution until $K$ SKUs have been added. The interchange heuristic starts with a given assortment and tests whether interchanging a SKU which is not in the assortment with a SKU in the assortment would increase revenue. Any revenue increasing interchanges are made as they are discovered. The process continues until a full pass over all possible interchanges discovers no revenue increasing interchanges. We apply the interchange heuristic both starting with the greedy assortment and starting with random assortments.

To find a portfolio of $L$ assortments and assignments of stores to assortments, we have two alternative heuristics, a forward and reverse greedy. In the forward greedy heuristic, we first apply the greedy and interchange heuristics to generate a single assortment for the chain. If $L>1$, we apply greedy and interchange to generate an assortment for each store and add as a second assortment, the store assortment that maximizes the revenue increase with stores optimally assigned to each assortment. This process continues until $L$ assortments have been chosen.

In the reverse greedy heuristic, we first apply greedy and interchange to generate an assortment for each store. If $L<m$, we identify the single store assortment to delete, that would minimize the revenue loss. We calculate the revenue loss from deleting an assortment by reassigning stores to their revenue maximizing assortment in the reduced set of assortments and calculating the loss in revenue due to the reassignment. At any point in the algorithm, we have a portfolio of $l$ assortments and $l$ store clusters defined by the assignment of stores to assortments. As long as $l>L$, we delete one assortment from this portfolio that leads to the least loss in revenue and reassign stores to the reduced set of assortments.

To evaluate the performance of the heuristics, we proved that if we use estimated, rather than actual prices for existing SKUs, the optimal solution in our applications has a special structure that allows us to find optimal assortments. We used this result to create optimal assortments for the 140 stores in the snack cake application and the 574 stores in the tires application, then applied greedy and found it generated solutions that were $97.2 \%$ and $98.5 \%$ of the optimal, respectively. We also tested the interchange heuristic, starting both with the greedy assortment and random assortments, but in no case found solutions that improved on the greedy assortment.

\section{Applications}

We worked with three retailers to apply our methodology, analyzing one product category for each of them: snack cakes for a regional convenience chain, tires for a national tire retailer and appearance chemicals for a major auto aftermarket parts retailer. Results for the three applications are summarized in Table 1. The entries in this table are described below.

\subsection{Description of Applications and SKU Attributes}

The regional convenience chain offered snack cakes in 60 flavors, two brands $\left\{B_{1}, B_{2}\right\}$, and several different package sizes in 140 stores. We restricted our analysis to the top 23 flavors that accounted for $95 \%$ of revenue. Although there were several different package sizes, what mattered from a consumer's 
perspective was whether the size was single-serve or family size, and hence we grouped sizes into Single Serve $(S)$ and Family Size $(F)$. Further, because the retailer advised us that brand shares and willingness to substitute varied by size, we combined brand and size to obtain a single attribute called Brand-Size, indexed 1 to 4 for $S B_{1}, S B_{2}, F B_{1}$ and $F B_{2}$ in order.

Thus there were 23 Flavor attribute levels, 4 Brand-Size attribute levels and 92 possible SKUs, of which 52 were being offered by the retailer in at least one store. The number of SKUs offered across stores varied between 24 and 52, and averaged 40.3. An internal market research study on the industry commissioned by the retailer showed that Flavor was the most important attribute for a consumer purchasing from this category. Hence, we assumed that the probability of substituting across flavors was negligible and could be set to 0 . The retailer also believed that there is negligible substitution between sizes $S$ and $F$, so this substitution was assumed to be 0 . The substitute for a particular brand-size is the other brand in the same size. We define $j(i)$ to be the brand-size that a customer might substitute to if $i$ is not in the assortment and set $j(1)=2, j(2)=1, j(3)=4$ and $j(4)=3$. We need to estimate the 23 flavor shares $f_{1 v}, 4$ brand-size shares $f_{2 b}$, and 4 substitution probability parameters $\pi_{12}, \pi_{21}, \pi_{34}$ and $\pi_{43}$.

Attributes for the tire study were brand, mileage warranty, and size. The retailer offered several nationally advertised brands that they believed were equivalent to the consumer, which we denote National $(\mathrm{N})$ and treat as one brand. They also offered three house brands of decreasing quality, which we denote as House 1 (H1), House 2 (H2) and House 3 (H3), where H1 is the highest quality and most expensive house brand. There were a large number of distinct mileage warranties offered, but some of these varied only slightly and hence were believed by the retailer to be equivalent to consumers. Therefore, we aggregated the mileage warranties into three levels of Low (15,000-40,000 miles), Medium (40,001-60,000 miles) and High (>60,000 miles), denoted L, M and H, respectively. We combined brand and warranty into a single attribute to account for interaction between these attributes (for example, national brands were always offered only with high or medium warranty, while $\mathrm{H} 3$ tires were offered only with low warranty) to identify the following six brand-warranty combinations: NH, NM, H1H, H2H, H2M, H3L. Sixty four distinct tire sizes were offered, resulting in $64 \times 6=384$ distinct possible tire SKUs that could be offered. This retailer carried 122 of these 384 possible SKUs in at least one of their stores. The number of SKUs offered across stores varied between 93 and 117, and averaged 105.2. The assortment offered also varied slightly across the stores, indicating some localization.

We were advised by the retailer that customers do not substitute across sizes. Table 2 is based on estimates provided by the Vice President of the tire category for the retailer and depicts the qualitative likelihood of substitution across brand-warranty levels. We let $\left\{\pi_{S}, \pi_{L}, \pi_{M}\right\}$ denote the substitution probabilities somewhat likely, likely and most likely.

The auto aftermarket parts retailer examines performance of each of their product categories once a year on a rotating schedule and considers changes in the assortment. We were asked in early May, 2009 to apply our methodology for the annual assortment reset for the appearance chemicals category, a category comprised of liquids and pastes for washing, waxing, polishing, protecting, etc. all surfaces of an auto, including the body, tires, wheels, windshield and other glass, and various interior surfaces. The retailer used a market research firm, NPD, that had assigned to each SKU in the appearance category the attributes (1) segment (defined by the surface of the car treated and what is done to that surface), (2) 9 brands and (3) 3 quality levels, denoted good, better or best, where 'good' is the lowest quality and 'best' is the highest. We appended package size, denoted small (S) or large (L), to the segment attribute 
to create 45 segment/size attribute levels. We combined brand and quality to create a second attribute, brand/quality. Because some brands did not offer all quality levels, there were 17, not 27 brand/quality combinations. In some cases there were two package sizes that differed slightly and were classified as $\mathrm{S}$ or L, so that two SKUs occupied the same cell of the attribute matrix. Consequently, the 160 SKUs currently offered corresponded to 130 cells of the $45 \times 17$ matrix of possible attribute levels. Of the $45 \times$ $17-130=635$ attribute combinations not carried by the retailer, only 24 were available in the market. No substitution parameters were used in the model for the following reason. If a brand-quality level were not offered for a product, it seemed likely that some of the demand for that brand-quality would transfer to several other brands. To estimate this effect we would have needed instances of different stores with varying numbers of brand-quality levels offered for the same product, and this data was not available.

For each of the applications, assortments varied somewhat across stores, although the retailers told us this was not the result of a systematic effort to localize assortments to store demand patterns, but rather due to varying store sizes. Typically, a small store would carry a subset of a large store assortment.

Given this, we developed a measure of assortment commonality that would indicate the extent of localization. For any pair of stores A and B, the overlap in assortment was computed as the ratio of the number of common SKUs to the minimum number of SKUs across the two stores. Note that this metric equals 1 if the assortment of one store is identical to or a subset of the other, indicating no localization. The average for all store pairs was $97.1 \%$ for Cakes, $94 \%$ for Tires and $96.4 \%$ for Appearance Chemicals, indicating that the extent of localization was minimal.

\subsection{Estimation}

Sales data for up to three time periods were used in the applications: fit, validation and implementation samples. For snack cakes, the fit sample was store-SKU sales from July - Dec 2005; the validation period was July - Dec 2007. For tires the fit and validation periods were July - Dec 2004 and Jan - June 2005 respectively. Results were implemented for tires and the impact on sales tracked for July - Dec 2005, the implementation sample. For appearance chemicals the fit period was May 2008 - April 2009. The results obtained in July 2009 were so compelling that the retailer decided to implement them in early January 2010 without validation. To evaluate the impact of implementation, we used store-SKU sales for the 27 week period 23 January - 8 July 2010.

We applied the estimation procedure described in 4.1 to Store-SKU sales data for the fit sample to estimate model parameters at each store for the three applications. This process worked except for 255 stores of the tire retailer where there was insufficient data to determine all 6 brand-warranty shares because there was no size in which brand-warranties H2M and H3L were both offered, making it impossible to identify the split of demand between H2M and H3L. Of these 255 stores, there were 52 stores where we could also not identify the share of H2M.We therefore regressed the H3L and H2M shares against median income for the 319 stores at which we could estimate all the parameters and used the regression estimate for these shares as needed. We then used MLE to estimate the remaining parameters.

To estimate prices of new SKUs not carried for snack cakes and tires, we used a hedonic regression as described in Section 4.2 to assign prices, with the $R^{2}$ values shown in Table 1 . We multiplied the estimated prices by a scale factor of 1.02 and 1.05 respectively, so as to equalize the estimated revenue at the chain level to the actual revenue, which will facilitate comparison of new optimized assortments with 
current revenue. The retailer of appearance chemicals sold branded products, so the prices of potential new SKUs were known.

\subsection{Validation}

We measured the overall estimation error across all stores, by computing the Sales-Weighted MeanAbsolute-Deviation (MAD) of estimated Store-SKU sales shares from actual Store-SKU sales shares, as given by $\frac{\sum_{s \in M} \sum_{j \in S^{s}}\left|\sum_{j}^{x_{j}^{s}}-F_{j}^{s}\left(S^{s}\right)\right| x_{j}^{s}}{\sum_{s \in M} \sum_{j \in S^{s}} \sum_{j}^{x_{j}^{s}}}$, where $S^{s}$ is the set of SKUs in the assortment for store $s$. We measure MAD in terms of sales shares because unit sales are significantly influenced by overall growth or shrinkage in the category, whereas sales shares are not. Moreover, our assortment choices are determined solely by sales shares, so these are the parameters important to our analysis.

We validated our results for the snack cake and tires applications using store-SKU sales for the validation periods defined above ${ }^{3}$. We used the fit estimated parameters to compute the share of sales for each SKU for the validation period, at the store and chain levels and compared it with the actual sales shares to obtain the MADs given in Table 1.

As discussed previously, there can be interaction between attributes, including size and brand-warranty in the tire application. One way to account for this interaction is to follow a latent class approach. We assume that there are several homogenous segments of sizes, and the brand-warranty shares vary across segments, but are the same within each segment. For example, a size segment could include tires that fit old cars, and the brand-warranty shares reflect this fact. To explore the feasibility of this approach, we estimated such a model for a subset of 20 stores, allowing for two size segments. We find that the sales-weighted MAD of sales share estimates was $15.3 \%$ as compared to $19.6 \%$ for the case of one size segment.

\subsection{Optimization}

We next applied the greedy heuristic described in Section 4.3 to compute optimized assortments at the chain and store levels, varying the number of SKUs in the assortment from 1 to $n$. Figure 1 shows the percentage of the maximum possible revenue if all SKUs were offered captured by the chain level assortment, as a function of $K$ as a percentage of $n$. Note that maximizing revenue for a given value of $K$ is equivalent to maximizing this percentage of maximum revenue captured.

For cakes and tires, the potential SKU set was all combinations of attributes. The appearance chemicals application sold branded products available in the market place, and hence there was a list of available products that we used in the optimization. As noted previously, this comprised SKUs currently offered and 24 potential new SKUs. Interestingly, while we were waiting to receive this list from the category manager, we did an analysis using all combinations of attributes which recommended adding a wax product for a leading brand. The category manager pointed out that the product did not exist in the market place, but then was shocked when the brand sales representative met with her several days later and told her they were planning to introduce exactly this product. So for retailers of branded products, it makes sense to analyze two cases: with SKUs that currently exist in the market place for assortment

\footnotetext{
${ }^{3}$ For the snack cakes validation we had data for only 54 of the 140 stores in the chain.
} 
update, and with a SKU set corresponding to all attribute combinations, so as to identify new product opportunities for suppliers and private label opportunities for the retailer.

All computations were carried out on the same computer with an Intel Core 2 Duo $2 \mathrm{GHz}$ processor. The computation times for estimation and greedy for each application are summarized in Table 1.

To quantify the potential improvement in revenue, we compared the assortments we generated for $L=1$ and $L=m$ to the current assortment. SKU count varied somewhat by store, so we compared the current assortment for store $s$ with SKU count $K_{s}$ to the greedy assortment for $K=K_{s}$.

\subsection{Findings}

Tables 3 - 7 give results of parameter estimation for the three applications. Table 2 for single serve, shows brand shares of $61 \%$ and $27 \%$, a more than 2 to 1 difference, while for family size, the two brands have equal shares. This shows the importance of our having combined brand and size into a single attribute, thus allowing for differences such as this. Similarly, as shown in Table 4, there is $89 \%$ substitution from brand 1 to 2 in family size, but only $18 \%$ in single serve. ${ }^{4}$ The retailer found this the most interesting finding of the study, as they believed substitution rates varied, but had previously no way to measure the exact rates.

The most interesting finding for the tire retailer was that, as shown in Table 5 , the demand share estimate for H3L is much higher than the sales share, while for H2M it is much lower. The reason for this appears to be that the retailer offered H3L in many fewer sizes than H2M; H3L is offered in only 15 of the 64 sizes, versus 52 sizes in which H2M is offered. But for those sizes where H3L and H2M are both offered, H3L outsells H2M by 40:1 on average, indicating that it is strongly preferred over H2M. The retailer offered H3L in fewer sizes because they preferred to sell the higher priced H2M and believed that their sales staff could convince customers to trade up to this tire. The substitution estimate of $45 \%$ shows that many customers did in fact trade up, and this explains the high sales share for H2M relative to its demand share. However, the $55 \%$ of the $61 \%$ of customers preferring H3L who did not substitute represents more than $34 \%$ of demand that was being lost due to the meager offering of H3L in the current assortment, suggesting that there was substantial opportunity to increase sales by re-assorting. We can see that offering H3L in only a few sizes hurts revenue. The average price of H3L and H2M in the sizes where both were offered was $\$ 28$ and $\$ 36$ respectively. Suppose that there were 100 consumers shopping the store and consider the two alternatives of offering H3L alone or H2M alone. Offering H2M would capture $(5 \% * 100+45 \% * 61 \% * 100) * \$ 36=\$ 1168$ in revenues while offering H3L would capture $(61 \% * 100) * \$ 28=\$ 1708$ implying $46 \%$ additional revenue.

Figure 2 shows that the estimated share of H3L at each store is negatively correlated and the share of $\mathrm{H} 2 \mathrm{H}$ and $\mathrm{H} 2 \mathrm{M}$ are positively correlated with median income level in the zip code in which the store is located, which is intuitive and provides confirming demographic evidence to support the reasonableness of our parameter estimates.

In the tire application it was feasible for the retailer to offer store-specific assortments, since the tires are not actually displayed at the store. But the snack cakes and appearance chemicals retailers thought it would be administratively too complex to have more than, respectively, 6 and 5 different assortments.

\footnotetext{
${ }^{4}$ Beswick and Isotta (2010), page 2, reports a very similar finding for an orange juice study. For the leading brand, only $21 \%$ are willing to substitute to another brands, but for the second brand, $85 \%$ are willing to substitute
} 
For snack cakes, we applied our assortment heuristics for $L=1,2, \ldots, 6 \cup m$, keeping $K=40$ for all stores $^{5}$. Table 8 shows revenue as a function of $L$. We note that complete localization increases revenue to $\$ 8.11$ million compared to the revenue of $\$ 7.38$ million for $L=1$. However, $76.7 \%$ of this increase can be achieved with just 6 different assortments, suggesting that a small amount of localization can have a big impact.

For appearance chemicals, we worked first on a 2,183 store 'warm up' case and then the full 3,236 store case, generating up to five different assortments. Table 9 shows estimated revenue increase for various cases. Based on results of the 2,183 store case, the retailer concluded that at most three store clusters would be used, so these were the cases run for the 3,236 store case. The retailer liked the two cluster solution and regarded cluster 1 as a base case that was representative of the chain as a whole and cluster two as a subset of stores differentiated, as shown in Table 7, by a higher level of tire related purchases, a preference for brand two, and a higher percentage of customers in a demographic the retailer called "urban/bilingual". This store segmentation was compelling for the retailer and agreed with more qualitative market research inputs they had received. Again we see that a small amount of localization produces most of the benefit.

In order to determine total market potential, we also ran an alternate scenario for Appearance Chemicals, expanding the potential SKU set to consist of all combinations of product attributes. This resulted in a chain optimal assortment lift of $15.2 \%$ and store optimal assortment lift of $19.7 \%$, compared to the values of $11.8 \%$ and $14.2 \%$ for the comparable cases using only SKUs available in the market.

\subsection{Implementation}

The tire and appearance chemicals retailers implemented a portion of our recommendations, which allowed us to assess how our methodology performed under a stringent implementation test.

Most of the products offered by the tire retailer were private label products. The retailer had relations with a number of suppliers from whom they could request production of a particular tire, but it was not always possible to find a supplier that would fulfill a given order. Usually the reason for declination was that the order was too small. Given this, the retailer asked us to optimize the assortment based on all attribute combinations, so they could take the resultant "shopping list" to the market to secure as many as possible of our recommended new tires. Our optimized assortment deleted 47 SKUs and replaced them with 47 new SKUs. Of these 47 new SKUs, the retailer could find suppliers for eleven, and these were added to the assortment in all stores, ten H3L SKUs and one H1H.

Estimating the change in revenue from the current to the implemented assortment was complicated because, in addition to adding eleven new SKUs to the assortment, the retailer deleted more than eleven SKUs in each store. The number of SKUs deleted varied somewhat by store but averaged 24 SKUs deleted. To achieve a fair comparison, in a store where Ns SKUs had been deleted and 11 added, we used the greedy heuristic to choose Ns-11 SKUs that were in the current assortment but not in the implemented assortment and added them to create a modified implementation assortment that had the same SKU count as the pre implementation assortment at each store and that was used in evaluation. We then used parameters estimated for the calibration, validation and implementation periods to estimate revenue for the current assortment, the modified implementation assortment and the two optimized

\footnotetext{
${ }^{5}$ We only report results obtained using the forward greedy heuristic as the results based on the reverse greedy heuristic were not significantly different.
} 
assortments. Table 10 gives revenue estimates and percentage improvement over the current baseline assortment for these periods, and shows a $5.8 \%$ revenue increase for the new assortment.

A 5.8\% improvement is significant. For example Canadian Tire reports achieving a 3-4\% annual revenue increase in existing stores during 2005-2009 and is targeting the same increase through 2012 as a result of all efforts, including assortment modification, to increase sales in existing stores (Canadian Tire Corporation Limited 2007).

The appearance chemicals retailer also adopted our recommendations, using the two cluster solution. In our recommended assortment, $20 \mathrm{SKUs}$ in cluster 1 with the lowest estimated revenue were replaced by 20 new attribute combinations. In cluster 2, 19 existing SKUs were replaced. The retailer used exactly the assignment of stores to clusters in our recommendations. They also adopted our recommendations on which attribute combinations to add to the assortment, although in some instances more than one SKU in the market corresponded to the same attribute combination, with the result that the number of SKUs added exceeded the number of attribute combinations added. Twenty two SKUs were added to cluster one and twenty five to cluster two. The choice of which SKUs to delete differed from our recommendations in the number of SKUs deleted and in which SKUs were deleted. Seventeen SKUs were deleted from cluster one and twenty three from cluster two. The choice of which SKUs to delete was guided by factors other than year to date revenue; for example, one car wash SKU was deleted due to a history of quality issues. In addition to assortment changes, their localization efforts included giving more prominent display and signage for tire products and brand 2 in the cluster 2 stores.

To evaluate the impact of these changes, we had available sales by cluster of the new assortment for the 27 week period January 1, 2010 - July 8, 2010, and of the previous assortment for a comparable period in 2009. In the discussion below, we refer to these as 2010 and 2009 sales, while recognizing they were for only a portion of these years. SKUs can be segmented into three groups: kept SKUs that were in both the 2009 and 2010 assortments, deleted SKUs that were in the 2009 assortment but not the 2010 and added SKUs that were in the 2010 assortment but not the 2009 assortment. To evaluate the impact of the assortment changes we compared 2010 kept plus added revenue to 2010 kept revenue plus an estimate of what 2010 revenue would have been for the 2009 SKUs deleted. Revenue is affected by a variety of factors other than assortment, including weather, the economy and competitive activity. We measured the impact of these other factors by the ratio of the 2010 to 2009 revenue for kept SKUs and estimated the 2010 revenue of the deleted SKUs as their 2009 revenue times this factor. We are thus comparing the new assortment revenue to an estimate of what the old assortment would have sold in 2010.

We needed to deal with the fact that more SKUs were added than deleted. Twenty two SKUs were added to cluster 1 vs. seventeen deleted and twenty five SKUs were added to cluster 2 vs. twenty three deleted. The retailer had a fixed amount of shelf space allocated to this category and accommodated the increase in SKU count by reducing the shelf space assigned to some of the existing SKUs. They therefore did not view the increase in SKU count as a cause for concern. Still, reducing the space for some existing SKUs might have caused greater stock outs, reducing revenue in a way we could not capture. Thus, to make a more rigorous evaluation of benefits, we used the twenty two and twenty five SKUs for clusters one and two, respectively, with lowest revenue in the 2009 evaluation period in estimating deletion revenue, thereby equalizing the add and delete counts.

The newly added SKUs were introduced some time after January 1, 2010 and hence were not on sale for the entire January 1 - July 8, 2010 period and moreover took some time to build to a steady state level 
of sales. Examining the weekly sales data of the added SKUs, we observed that it took them 7 weeks to achieve a steady steady sales rate. Hence, we used added SKU revenue for weeks $8-27$ scaled by $27 / 20$ as our estimate of added SKU revenue for the period January 1 - July 8, 2010.

The result of these calculations showed a $3.6 \%$ revenue increase due to the revised assortment. In addition, there may have been some improvement due to the localized product display and signage in cluster two stores that we were not able to measure. The retailer's appearance chemicals team agreed with our assessment of benefits and believed that the re-assortment exercise had been a success. We note that a $3.6 \%$ revenue increase is large relative to the usual impact of all things retailers do to increase same store sales. For example, in their annual reports, four leading auto parts retailers (Advance Auto Parts, Auto Zone, O'Reilly, and Pep Boys) reported comparable store sales increases over 2006-2010 that averaged $1.9 \%$.

\subsection{Forecasting New SKUs}

One new SKU was added to the snack cakes assortment in the July-December 2007 validation period, Butterscotch in Brand 2 Single Serve. Eleven SKUs were added to the tire assortment and 25 to the appearance chemicals assortment during the implementation periods. Table 1 shows the MAPE of all new SKU forecasts. We note that these values compare favorably to the $30.7 \%$ MAPE for chain sales of two new SKUs reported by Fader and Hardie 1996.

Sources of forecast error affecting the fit, validation and implementation samples include random fluctuation in sales and the approximation of representing SKU shares as the product of attribute shares. An additional source of error for the validation and implementation samples are demand trends over time. For example, the demand for a particular tire initially increases over time as cars that use the tire age and need replacement tires, but eventually declines as those cars become old enough that they begin to exit the population. Price changes can also impact demand patterns in any application. Table 11 shows how changes in relative prices across tire brand-warranties relates to systematic changes in their demand shares over time. In particular, it is striking that as the price difference between H2M and H3L narrowed from $43.7 \%$ to $41.9 \%$ to $22.9 \%$ over the three periods, the demand split between these two brand-warranties shifted from $7 \% / 70 \%$ to $29 \% / 27 \%$.

\section{Factors Influencing Localization Revenue Lift}

The Localization Lift, defined as the revenue increase from using store specific assortments vs. a single assortment for the chain was $12.2 \%, 5.8 \%$ and $2.4 \%$ for the snack cakes, tires and appearance chemicals examples described in the previous sections. As we sought to understand what features of the problem data cause these differences in Localization Lift, our first thought was that Localization Lift must be driven by demand variation across stores. We thus calculated a coefficient of demand variation (COV) defined as $\sqrt{\sum_{i \in N} \sigma_{i}^{2}} / \sum_{i \in N} \mu_{i}$, where $\mu_{i}$ and $\sigma_{i}$ denote the mean and standard deviation of revenue shares of SKU $i$ across all stores. COV for snack cakes, tires and appearance chemicals was $17 \%, 11 \%$ and $10 \%$ respectively. These values shed some light on variation in Localization Lift in that the highest COV matches the highest lift, for snack cakes, but leave open the drivers of variation in Localization Lift between tires and appearance chemicals, where the Localization Lift varies by a factor of three while the COV's are nearly equal. 
To better understand this issue, we examined the data more closely and made two observations. First, SKUs can be segmented into three groups: (1) those with such high demand that they were carried in every store optimal assortment, (2) those with such low demand that they were in no store optimal assortment and (3) the remainder. While there may be substantial variation in demand across stores for the first groups, none of this variation impacts Localization Lift.

For example, in the case of appearance chemicals, we see in Table 7 that the best selling segment-size for the chain is Tire Dressings/Shines TRIGGER L. Table 12 shows substantial difference in the sales rate between two stores for Tire Dressings/Shines TRIGGER L. The best selling single SKU in this segment-size, as defined by brand and quality level, accounted for 5.5\% of revenue in Store A and $16.6 \%$ in Store B, a 3.3 to 1 difference. Yet even though the SKU sold much worse in Store A, with a revenue share of $5.5 \%$, it clearly made sense to have this SKU in a revenue maximizing assortment for Store A, and hence this difference in sales rate had no impact on the Localization Lift.

Secondly, we noticed substantial variation in the breadth of assortment carried, from 40 out of 92 possible SKUs for snack cakes to 130 out of 154 possible SKUs for appearance chemicals. A broader assortment means that a single chain optimal assortment captures a greater fraction of potential demand, leaving less room for improvement from assortment localization.

The example in Table 13 is designed to illustrate how these patterns can occur. The example compares $\mathrm{COV}$ and Localization Lift for two demand cases, and within each case, for $K$ equal to 3 or 4 . The price of all SKUs is $\$ 1$, so unit demand and revenue are the same. Moreover, total demand is equal for the two stores, so there is no distinction between demand units and demand share. We also assume there is no substitution. The variance column gives the variance in demand across the two stores for each SKU. $\mathrm{COV}$, as defined above is the square root of total variance divided by total demand.

In all cases the chain optimal assortment is SKUs 1 through K. For $K=3$, the optimal assortment for store 1 is SKUs 1, 2 and 3 and for store 2, SKUs 1, 2 and 4. For $K=4$, SKUs 1 to 4 are an optimal assortment for both stores.

For $K=3$, note that Case 1 has the highest COV but the lowest Localization Lift. This happens because almost all of the inter store demand variation occurs for SKUs that are in either both store optimal assortments or neither and hence none of this variation impacts Localization Lift. By contrast, in Case 2, all of the inter store demand variation occurs for SKUs 3 and 4, the very SKUs that differ in the store optimal assortments. For $\mathrm{K}=4$, the lift in both cases drops to 0 , demonstrating the impact that breath of assortment can have on lift.

Motivated by what we saw in the data for the three applications and by the features demonstrated by the example in Table 13, we defined two additional metrics which we hypothesize would be correlated with Localization Lift. COV Select $(\mathrm{K})$ is the coefficient of variation for SKUs which are in some but not all store optimal assortments. This metric is a function of $\mathrm{K}$ because store optimal assortments depend on K. We re-label our original coefficient of variation metric as COV - All to emphasize its difference with COV - Select (K).

We also define Chain Optimal Share (K) to be the share of the total potential revenue $\sum_{s \in M} \sum_{i \in N} p_{i} D_{i}^{s}$ achieved by a single, chain optimal assortment with K SKUs. So far we have not discussed the impact of substitution. We simply note that an increase in willingness to substitute increases Chain Optimal Share (K) and thus decreases Localization Lift.

Table 14 gives Localization Lift and the three metrics for the three applications. We observe that the 
highest Localization Lift for cakes can be explained by the low value of Chain Optimal (K) and high values of COV - All and COV Select $(\mathrm{K})$. We also note that the difference in Localization Lift between tires and appearance chemicals can be explained by a high value of Chain Optimal Share $(\mathrm{K})$ and a relatively low value of COV - Select (K) for appearance chemicals.

We also used the solutions to the three applications for $K$ varying from 1 to $n$ to create Figure 3 showing Localization Lift versus Percent of Maximum Total SKUs in the Assortment and Chain Optimal Share (K). We note that Localization Lift varies with Chain Optimal Share (K) as we have hypothesized. The dip in the lift index initially in Figure 3 is because the top SKUs at the chain level have different rank ordering across stores.

To further investigate the effect of demand variation and share captured by the chain optimal assortment on localization lift, we used the existing data to create 100 additional problem instances for each of the three applications by randomizing (a) the number of stores in the chain, (b) the actual stores sampled and (c) K, the maximum number of SKUs allowed in the assortment. Table 15 gives the range used for each application in randomly generating store count and K. We then calculated Localization Lift, COV - All, COV - Select and Chain Optimal Share (K) and regressed Localization Lift against the three dependent variables; the results are summarized in Table 15. The regression results confirm our hypothesis that Localization Lift depends mainly on Chain Optimal Share (K) and COV - Select, which are highly significant, and not on COV - All, which is insignificant.

\section{Conclusions}

We have formulated a process for finding optimal assortments, comprised of a demand model, and estimation approach and heuristics for choosing assortments. We have applied this process to real data from three applications and shown that the approach produces accurate forecasts for new SKUs. Our recommendations were implemented in two of the cases. We measured the impact based on actual sales and found the assortment revisions had produced revenue increases of $5.8 \%$ and $3.6 \%$, which are significant relative to typical comparable store increases in these product segments.

We note the following observations from this research.

1. Forecast accuracy for new SKUs compared favorably to prior research in Fader and Hardie (1996) and was adequate to achieve significant benefits in implementation. Nonetheless, the errors were great enough to reduce the revenue increase by about half from the fit to validation samples, so improving forecast accuracy would be a useful focus of future research.

2. Sales is not true demand, but demand distorted by the assortment offered. We do not see demand for SKUs not offered, and the sales of SKUs offered may be increased above true demand due to substitution. The impact of these effects can be significant. In the tire application, the lowest price brand-warranty level had a demand share of $61 \%$ but a sales share of only $5 \%$, because the retailer offered the lowest price brand-warranty in few sizes. Adding more of this brand warranty to the assortment was a big source of the revenue increase attained.

3. Substitution can be measured, can vary significantly and have a major impact on the optimal assortment. In the snack cakes example, in the family size, the probability of substituting from 
Brand 1 to Brand 2 was $89 \%$, versus only a $22 \%$ probability of substituting from Brand 2 to Brand 1. This resulted in a complete replacement of Brand 1 by Brand 2 in family size of the optimized assortment.

4. We were able to use demographic data to confirm our parameter estimates in the tire and appearance chemicals examples. In particular, the share of the lowest price tire and unwillingness to substitute up to a higher price tire were correlated with median income in the store area. In some instances, we also used demographic data to assist in estimating parameters.

5. The benefit from localizing assortments by store varied considerably, from $2 \%$ to $12 \%$. We showed that this difference is not only driven by the coefficient of demand variation across stores, but also by the percent of maximum revenue captured by a chain optimal assortment and by the coefficient of variation in demand for those SKUs that are in some, but not all store optimal assortments.

6. A limited amount of localization can capture most of the benefits of maximum localization. In the snack cakes example, going from 1 assortment to 6 provided $77 \%$ of the benefit as going from 1 assortment to 140 assortments.

7. There may be interaction between attribute levels not captured by our demand model. In the case of tires, the demand for the least expensive brand-warranty level will be higher for a size tire that goes on an older, inexpensive car than for a tire that goes on a new, luxury car. We showed that this could be incorporated into our approach through latent class analysis.

\section{Acknowledgments}

We thank Professors Abba Krieger and David Bell from the Wharton School for numerous valuable comments and helpful suggestions with our research and Paul Beswick, Partner and Head, Oliver Wyman North American Retail Practice, Robert DiRomualdo, former CEO, Borders Group, Kevin Freeland, COO, Advance Auto, Matthew Hamory, Principal, Oliver Wyman North American Retail Practice, Herbert Kleinberger, Principal, ARC Consulting, Chris Morrison, Senior VP of Sales, Americas, Tradestone, Robert Price, Chief Marketing Officer, CVS, and Cheryl Sullivan, vice president of product management, Revionics, Inc. for helpful discussions on assortment planning practice. We also thank Carol Jensen, Tamara H. Jermyn, and Amanda O'Brien from Wawa, Inc. for their support. The financial support of the Wharton School Fishman Davidson Center for Service and Operations Management is gratefully acknowledged. 


\section{Tables}

Table 1: Summary of key values for the three applications

\begin{tabular}{|c|c|c|c|}
\hline & \multicolumn{3}{|c|}{ Application } \\
\hline & Snack Cakes & Tires & Appearance Chemicals \\
\hline \multicolumn{4}{|c|}{ Category Parameters } \\
\hline $\mathrm{n}-$ possible SKU count & 92 & 384 & 154 \\
\hline K - average number of SKUs in an assortment & 40 & 105 & 130 \\
\hline m - store count & 140 & 574 & 3,236 \\
\hline \multicolumn{4}{|c|}{ Demand Forecasts } \\
\hline Fit MAD, Chain Level & $6.2 \%$ & $4.5 \%$ & $8.5 \%$ \\
\hline Fit MAD, Store Level & $16.4 \%$ & $13.6 \%$ & $19.3 \%$ \\
\hline Validation MAD, Chain Level & $25.8 \%$ & $21.1 \%$ & \\
\hline Validation MAD, Store Level & $40.1 \%$ & $38.2 \%$ & \\
\hline Price Regression $R^{2}$ & $85.5 \%$ & $96.3 \%$ & \\
\hline \multicolumn{4}{|c|}{ Estimated Revenue Increase (Based on Fit Sample) } \\
\hline Chain Optimal Assortment & $29.2 \%$ & $30.1 \%$ & $11.8 \%$ \\
\hline Store optimal assortment & $41.4 \%$ & $35.9 \%$ & $14.2 \%$ \\
\hline Implementation & & $5.8 \%$ & $3.6 \%$ \\
\hline Number of New SKUs Forecasted & 1 & 11 & 25 \\
\hline New SKU Forecast MAPE & $16.2 \%$ & $19.1 \%$ & $28.7 \%$ \\
\hline \multicolumn{4}{|c|}{ Computation Time (Average per Store) } \\
\hline Estimation & $7.2 \mathrm{~s}$ & $13.3 \mathrm{~s}$ & $5 \mathrm{~s}$ \\
\hline Greedy & $2.2 \mathrm{~s}$ & $6.5 \mathrm{~s}$ & $1.4 \mathrm{~s}$ \\
\hline
\end{tabular}

Table 2: Tires: Management's estimate of the most likely substitution probabilities

\begin{tabular}{|c|c|c|c|c|c|c|}
\hline From & $\begin{array}{l}\text { To } \\
\text { NH }\end{array}$ & NM & $\mathrm{H} 1 \mathrm{H}$ & $\mathrm{H} 2 \mathrm{H}$ & $\mathrm{H} 2 \mathrm{M}$ & H3L \\
\hline $\mathrm{NH}$ & 1 & $S$ & $S$ & $S$ & 0 & 0 \\
\hline $\mathrm{NM}$ & $L$ & 1 & $S$ & $S$ & 0 & 0 \\
\hline $\mathrm{H} 1 \mathrm{H}$ & 0 & 0 & 1 & $L$ & $S$ & 0 \\
\hline $\mathrm{H} 2 \mathrm{H}$ & 0 & 0 & $S$ & 1 & $S$ & 0 \\
\hline $\mathrm{H} 2 \mathrm{M}$ & 0 & 0 & $S$ & $L$ & 1 & 0 \\
\hline H3L & 0 & 0 & 0 & 0 & $M$ & 1 \\
\hline \multicolumn{7}{|c|}{$S=$ Somewhat Likely, L $=$ Likely, $M=$ Most Likely } \\
\hline
\end{tabular}


Table 3: Snack Cakes: Demand Share Estimates (Averaged across Stores)

\begin{tabular}{ccc}
\hline Brand Size & Sales Share & $\begin{array}{c}\text { Demand } \\
\text { Share }\end{array}$ \\
\hline$B_{1} S$ & $67 \%$ & $61 \%$ \\
$B_{2} S$ & $24 \%$ & $27 \%$ \\
$B_{1} F$ & $4 \%$ & $6 \%$ \\
$B_{2} F$ & $5 \%$ & $6 \%$ \\
\hline
\end{tabular}

Table 5: Tires: Demand Share Estimates (Averaged across Stores)

\begin{tabular}{lcc}
\hline $\begin{array}{l}\text { Brand } \\
\text { Warranty }\end{array}$ & $\begin{array}{c}\text { Sales } \\
\text { Share }\end{array}$ & $\begin{array}{c}\text { Demand } \\
\text { Share }\end{array}$ \\
\hline NH & $1 \%$ & $4 \%$ \\
NM & $1 \%$ & $3 \%$ \\
H1H & $3 \%$ & $4 \%$ \\
H2H & $26 \%$ & $24 \%$ \\
H2M & $45 \%$ & $5 \%$ \\
H3L & $24 \%$ & $61 \%$ \\
\hline
\end{tabular}

Table 4: Snack Cakes: Substitution Probability Estimates (Averaged across Stores)

\begin{tabular}{ccccc}
\hline & $B_{1} S$ & $B_{2} S$ & $B_{1} F$ & $B_{2} F$ \\
\hline$B_{1} S$ & 1 & $18 \%$ & 0 & 0 \\
$B_{2} S$ & $26 \%$ & 1 & 0 & 0 \\
$B_{1} F$ & 0 & 0 & 1 & $89 \%$ \\
$B_{2} F$ & 0 & 0 & $22 \%$ & 1 \\
\hline
\end{tabular}

Table 6: Tires: Substitution Probability Estimates (Averaged across Stores)

\begin{tabular}{lc}
\hline Substitution Likelihood & Probability \\
\hline Somewhat Likely & $2 \%$ \\
Likely & $6 \%$ \\
Most Likely & $45 \%$ \\
\hline
\end{tabular}

Table 7: Appearance Chemicals: Cluster Statistics for Top 25 SKUs for Appearance Chemicals

\begin{tabular}{|c|c|c|c|c|c|c|c|}
\hline \multicolumn{8}{|c|}{ Revenue Distribution by Product type - package size, Quality and Brand } \\
\hline Segment - package size & Cluster 1 & Cluster 2 & Total & Quality & Cluster 1 & Cluster 2 & Total \\
\hline Tire Dressings / Shines TRIGGER L & $7.1 \%$ & $12.6 \%$ & $8.8 \%$ & Good & $12 \%$ & $14 \%$ & $13 \%$ \\
\hline Multi-Purpose Protectants S & $7.2 \%$ & $8.4 \%$ & $7.6 \%$ & Better & $60 \%$ & $67 \%$ & $62 \%$ \\
\hline Washes L & $7.4 \%$ & $6.6 \%$ & $7.1 \%$ & Best & $28 \%$ & $20 \%$ & $25 \%$ \\
\hline Tire Dressings / Shines AEROSOL S & $5.0 \%$ & $7.4 \%$ & $5.8 \%$ & Total & $100 \%$ & $100 \%$ & $100 \%$ \\
\hline Tire Cleaners TRIGGER L & $4.6 \%$ & $7.4 \%$ & $5.5 \%$ & & & & \\
\hline Liquid Wax S & $5.5 \%$ & $3.6 \%$ & $4.9 \%$ & Brand & Cluster 1 & Cluster 2 & Total \\
\hline Wash and Wax S & $4.6 \%$ & $4.4 \%$ & $4.6 \%$ & Brand 1 & $18 \%$ & $20 \%$ & $19 \%$ \\
\hline Washes S & $4.9 \%$ & $3.8 \%$ & $4.6 \%$ & Brand 2 & $7 \%$ & $11 \%$ & $8 \%$ \\
\hline Multi-Purpose Protectants L & $4.0 \%$ & $3.8 \%$ & $4.0 \%$ & Brand 3 & $6 \%$ & $6 \%$ & $6 \%$ \\
\hline Tire Foams (multi-purpose) AEROSOL S & $3.1 \%$ & $4.1 \%$ & $3.4 \%$ & Brand 4 & $19 \%$ & $16 \%$ & $18 \%$ \\
\hline Carpet / Upholstery Cleaners AEROSOL S & $3.2 \%$ & $3.0 \%$ & $3.1 \%$ & Brand 5 & $12 \%$ & $7 \%$ & $10 \%$ \\
\hline Spray Wax S & $3.0 \%$ & $2.6 \%$ & $2.9 \%$ & Brand 6 & $6 \%$ & $7 \%$ & $7 \%$ \\
\hline Paste Wax S & $3.0 \%$ & $2.2 \%$ & $2.8 \%$ & Brand 7 & $2 \%$ & $2 \%$ & $2 \%$ \\
\hline Wheel Care / Cleaner. ALL TRIGGER S & $2.7 \%$ & $2.8 \%$ & $2.7 \%$ & Brand 8 & $11 \%$ & $10 \%$ & $11 \%$ \\
\hline Spray Detailers L & $3.0 \%$ & $2.1 \%$ & $2.7 \%$ & Brand 9 & $19 \%$ & $21 \%$ & $19 \%$ \\
\hline Leather Cleaners / Conditioners TRIGGER S & $2.7 \%$ & $2.0 \%$ & $2.5 \%$ & Total & $100 \%$ & $100 \%$ & $100 \%$ \\
\hline Wheel Care / Cleaner. ALL TRIGGER L & $2.4 \%$ & $2.5 \%$ & $2.4 \%$ & & & & \\
\hline Rubbing / Polishing Compounds S & $2.1 \%$ & $1.5 \%$ & $1.9 \%$ & & & & \\
\hline Tire Dressings / Shines BOTTLE GEL S & $1.9 \%$ & $1.2 \%$ & $1.7 \%$ & & \multicolumn{3}{|c|}{ Demographic statistics } \\
\hline Scratch Removers S & $1.8 \%$ & $1.1 \%$ & $1.6 \%$ & & Cluster 1 & Cluster 2 & Total \\
\hline Rubbing / Polishing Compounds L & $1.9 \%$ & $0.9 \%$ & $1.6 \%$ & Income Index & 0.95 & 0.80 & 0.90 \\
\hline Glass Cleaners TRIGGER L & $1.5 \%$ & $1.6 \%$ & $1.5 \%$ & $\%$ Suburban & $85 \%$ & $62 \%$ & $78 \%$ \\
\hline Plastic / Lens Cleaners, Polishes \& Repair S & $1.6 \%$ & $1.3 \%$ & $1.5 \%$ & $\%$ Urban/bilingual & $16 \%$ & $42 \%$ & $24 \%$ \\
\hline Glass Cleaners AEROSOL S & $1.6 \%$ & $1.4 \%$ & $1.5 \%$ & Store count & 2263 & 973 & $15 \%$ \\
\hline
\end{tabular}


Table 8: Snack Cakes: Impact of Amount of Localization on Revenue

\begin{tabular}{cc}
\hline$L$ & Revenues (\$ million) \\
\hline 1 & 7.38 \\
2 & 7.62 \\
3 & 7.75 \\
4 & 7.86 \\
5 & 7.92 \\
6 & 7.94 \\
$\ldots$ & $\ldots .11$ \\
$m=140$ & 8.11 \\
\hline
\end{tabular}

Table 9: Appearance Chemicals: Estimated Revenue Increases (\%) vs. Number of Store Clusters

\begin{tabular}{ccc}
\hline $\begin{array}{c}\text { No. of } \\
\text { Clusters }\end{array}$ & $\begin{array}{c}2183 \text { Stores } \\
\text { (warm up) }\end{array}$ & $\begin{array}{c}3236 \text { Stores } \\
\text { (implementation) }\end{array}$ \\
\hline 1 & 14.1 & 11.8 \\
2 & 14.4 & 11.9 \\
3 & 14.6 & 12.0 \\
5 & 14.9 & \\
\hline
\end{tabular}

Table 10: Tires: Revenue Estimates for Current, Implemented and Optimized Assortments (percentage improvement over current revenues given in parenthesis)

\begin{tabular}{cccc}
\hline Revenues (\$\$ million) & \multicolumn{3}{c}{ Parameter Estimates Used } \\
Assortment & Jul 04 - Dec 04 & Jan 05 - Jun 05 & Jul 05 - Dec 05 \\
\hline Current Assortment (Jul 04 - Dec 04) & 80.2 & 74.9 & 72.3 \\
Modified Implementation (Jul 05 - Dec 05) & $90.7(13.1)$ & $84.7(13.1)$ & $76.5(5.8)$ \\
Recommended Optimal Assortment, Chain & $104.1(29.8)$ & $94.3(25.9)$ & $81.4(12.6)$ \\
Recommended Optimal Assortment, Store & $108.2(34.9)$ & $99.2(32.4)$ & $83.6(15.6)$ \\
\hline
\end{tabular}

Table 11: Tires: Price Changes and Impact on Demand Shares for a Representative Store

\begin{tabular}{|c|c|c|c|}
\hline \multicolumn{4}{|c|}{ Share of Demand (Price in $\$ \$$ ) } \\
\hline $\begin{array}{c}\text { Brand } \\
\text { Warranty }\end{array}$ & $\begin{array}{c}\text { Jul - Dec } \\
2004\end{array}$ & $\begin{array}{c}\text { Jan - Jun } \\
2005\end{array}$ & $\begin{array}{c}\text { Jul - Dec } \\
2005\end{array}$ \\
\hline $\mathrm{NH}$ & $2(73.9)$ & $5(69.7)$ & $8(77.6)$ \\
\hline NM & $2(58.9)$ & $3(51.4)$ & $6(50.9)$ \\
\hline $\mathrm{H} 1 \mathrm{H}$ & $3(59.8)$ & $10(61.1)$ & $8(58.6)$ \\
\hline $\mathrm{H} 2 \mathrm{H}$ & $16(49.6)$ & $21(53.5)$ & $22(56.5)$ \\
\hline $\mathrm{H} 2 \mathrm{M}$ & $7(43.3)$ & $10(45.7)$ & $29(46.5)$ \\
\hline H3L & $70(30.1)$ & $51(32.2)$ & $27(37.9)$ \\
\hline $\begin{array}{l}\text { H2M-H3L \% } \\
\text { Price Difference }\end{array}$ & 43.7 & 41.9 & 22.9 \\
\hline
\end{tabular}


Table 12: Statistics for Stores with Maximum and Minimum Percent Suburban

Revenue Distribution by Product type - package size, Quality and Brand

\begin{tabular}{|c|c|c|c|c|c|}
\hline Segment - package size & Store A & Store B & Quality & Store A & Store B \\
\hline Tire Dressings / Shines TRIGGER L & $5.5 \%$ & $16.6 \%$ & Good & $13 \%$ & $18 \%$ \\
\hline Multi-Purpose Protectants S & $5.3 \%$ & $10.6 \%$ & Better & $57 \%$ & $66 \%$ \\
\hline Washes L & $7.8 \%$ & $8.5 \%$ & Best & $31 \%$ & $16 \%$ \\
\hline Tire Dressings / Shines AEROSOL S & $4.6 \%$ & $8.8 \%$ & Total & $100 \%$ & $100 \%$ \\
\hline Tire Cleaners TRIGGER L & $6.5 \%$ & $5.3 \%$ & & & \\
\hline Liquid Wax $\mathrm{S}$ & $8.1 \%$ & $2.3 \%$ & Brand & Store A & Store B \\
\hline Wash and Wax S & $2.8 \%$ & $5.0 \%$ & Brand 1 & $16 \%$ & $18 \%$ \\
\hline Washes S & $4.5 \%$ & $3.4 \%$ & Brand 2 & $6 \%$ & $15 \%$ \\
\hline Multi-Purpose Protectants L & $4.8 \%$ & $3.2 \%$ & Brand 3 & $5 \%$ & $6 \%$ \\
\hline Tire Foams (multi-purpose) AEROSOL S & $3.4 \%$ & $3.0 \%$ & Brand 4 & $16 \%$ & $12 \%$ \\
\hline Carpet / Upholstery Cleaners AEROSOL S & $2.3 \%$ & $3.2 \%$ & Brand 5 & $17 \%$ & $4 \%$ \\
\hline Spray Wax S & $4.1 \%$ & $2.0 \%$ & Brand 6 & $7 \%$ & $10 \%$ \\
\hline Paste Wax S & $2.2 \%$ & $3.0 \%$ & Brand 7 & $2 \%$ & $2 \%$ \\
\hline Wheel Care / Cleaner. ALL TRIGGER S & $2.3 \%$ & $2.8 \%$ & Brand 8 & $10 \%$ & $10 \%$ \\
\hline Spray Detailers L & $5.1 \%$ & $1.2 \%$ & Brand 9 & $22 \%$ & $23 \%$ \\
\hline Leather Cleaners / Conditioners TRIGGER S & $3.2 \%$ & $1.2 \%$ & Total & $100 \%$ & $100 \%$ \\
\hline Wheel Care / Cleaner. ALL TRIGGER L & $3.4 \%$ & $1.0 \%$ & & & \\
\hline Rubbing / Polishing Compounds S & $0.5 \%$ & $2.5 \%$ & & \multicolumn{2}{|c|}{ Demographic statistics } \\
\hline Tire Dressings / Shines BOTTLE GEL S & $1.5 \%$ & $1.8 \%$ & & Store A & Store B \\
\hline Scratch Removers S & $1.4 \%$ & $1.6 \%$ & Income Index & 0.98 & 0.88 \\
\hline Rubbing / Polishing Compounds L & $2.0 \%$ & $1.1 \%$ & $\%$ Suburban & $98 \%$ & $8 \%$ \\
\hline Glass Cleaners TRIGGER L & $2.0 \%$ & $1.1 \%$ & $\%$ Urban/bilingual & $1 \%$ & $91 \%$ \\
\hline Plastic / Lens Cleaners, Polishes \& Repair S & $1.5 \%$ & $1.3 \%$ & Location & Marietta, $\mathrm{OH}$ & Lithonia, GA \\
\hline
\end{tabular}

Table 13: Example to Illustrate Drivers of Localization

\begin{tabular}{|c|c|c|c|c|c|c|}
\hline & \multicolumn{3}{|c|}{ Case 1} & \multicolumn{3}{|c|}{ Case 2} \\
\hline & \multicolumn{2}{|c|}{ Demand } & \multirow[b]{2}{*}{ Variance } & \multicolumn{2}{|c|}{ Demand } & \multirow[b]{2}{*}{ Variance } \\
\hline SKU & Store 1 & Store 2 & & Store 1 & Store 2 & \\
\hline 1 & 50 & 100 & 1250 & 75 & 75 & 0 \\
\hline 2 & 100 & 50 & 1250 & 75 & 75 & 0 \\
\hline 3 & 40 & 50 & 50 & 50 & 0 & 1250 \\
\hline 4 & 50 & 40 & 50 & 0 & 50 & 1250 \\
\hline 5 & 40 & 0 & 800 & 25 & 25 & 0 \\
\hline 6 & 0 & 40 & 800 & 25 & 25 & 0 \\
\hline 7 & 40 & 0 & 800 & 25 & 25 & 0 \\
\hline 8 & 0 & 40 & 800 & 25 & 25 & 0 \\
\hline 9 & 40 & 0 & 800 & 25 & 25 & 0 \\
\hline 10 & 0 & 40 & 800 & 25 & 25 & 0 \\
\hline 11 & 40 & 0 & 800 & 25 & 25 & 0 \\
\hline 12 & 0 & 40 & 800 & 25 & 25 & 0 \\
\hline Totals & 400 & 400 & 9000 & 400 & 400 & 2500 \\
\hline $\mathrm{COV}$ & & & $11.9 \%$ & & & $6.4 \%$ \\
\hline \multirow[t]{2}{*}{$\mathbf{K}$} & Chain & Localized & Localization & Chain & Localized & Localization \\
\hline & Optimal & Optimal & Lift & Optimal & Optimal & Lift \\
\hline 3 & 390 & 400 & $2.6 \%$ & 350 & 400 & $14.3 \%$ \\
\hline 4 & 480 & 480 & 0 & 400 & 400 & \\
\hline
\end{tabular}


Table 14: Explaining Localization Lift

\begin{tabular}{lcccc}
\hline Category & Lift & $\begin{array}{c}\text { COV - } \\
\text { All }\end{array}$ & $\begin{array}{c}\text { COV - } \\
\text { Select } \\
(\mathbf{K})\end{array}$ & $\begin{array}{c}\text { Chain } \\
\text { Optimal } \\
\text { Share }\end{array}$ \\
\hline Cakes & 0.122 & 0.17 & 0.23 & 0.71 \\
Tires & 0.058 & 0.11 & 0.12 & 0.80 \\
Appearance & 0.024 & 0.10 & 0.10 & 0.93 \\
Chemicals & & & & \\
\hline
\end{tabular}

Table 15: Regression of Localization Lift

\begin{tabular}{lccl}
\hline Coefficient & Cakes & Tires & $\begin{array}{l}\text { Appearance } \\
\text { Chemicals }\end{array}$ \\
\hline (Intercept) & $0.147^{* * *}$ & $0.113^{* * *}$ & $0.186^{* * *}$ \\
Chain Optimal Share & $-0.095^{* * *}$ & $-0.166^{* * *}$ & $-0.157^{* * *}$ \\
COV Select & $0.045^{*}$ & $0.475^{* * *}$ & $0.339^{* * *}$ \\
COV All & -0.024 & 0.232 & -0.000 \\
\hline Simulation Details & & & \\
Minimum K & 10 & 10 & 5 \\
Maximum K & 40 & 100 & 40 \\
Minimum Stores & 10 & 20 & 80 \\
Maximum Stores & 30 & 60 & 240 \\
\# of Instances Simulated & 100 & 100 & 100 \\
\hline
\end{tabular}

\section{$9 \quad$ Figures}

Figure 1: Revenue vs. Percent of Maximum Possible SKUs in the Assortment

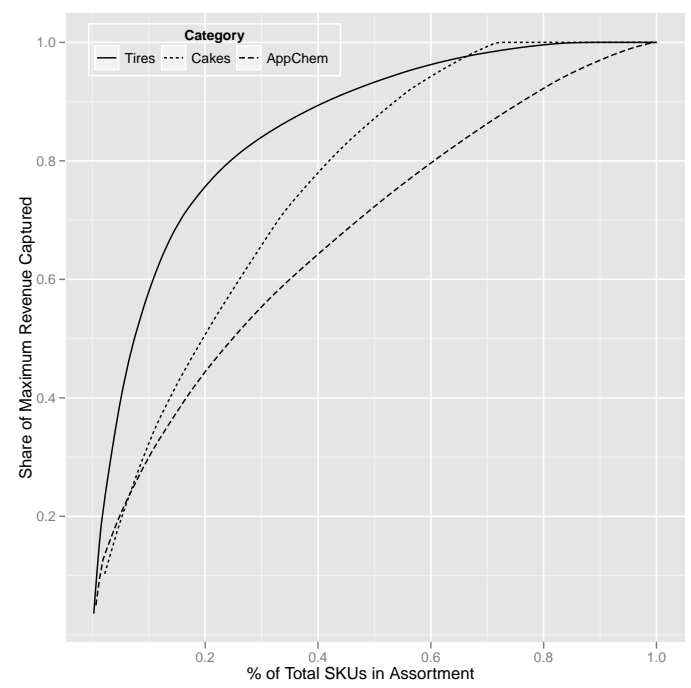

Figure 2: Tires: Share of $H 3 L(H 2 H, H 2 M)$ is Negatively (Positively) Correlated with Income

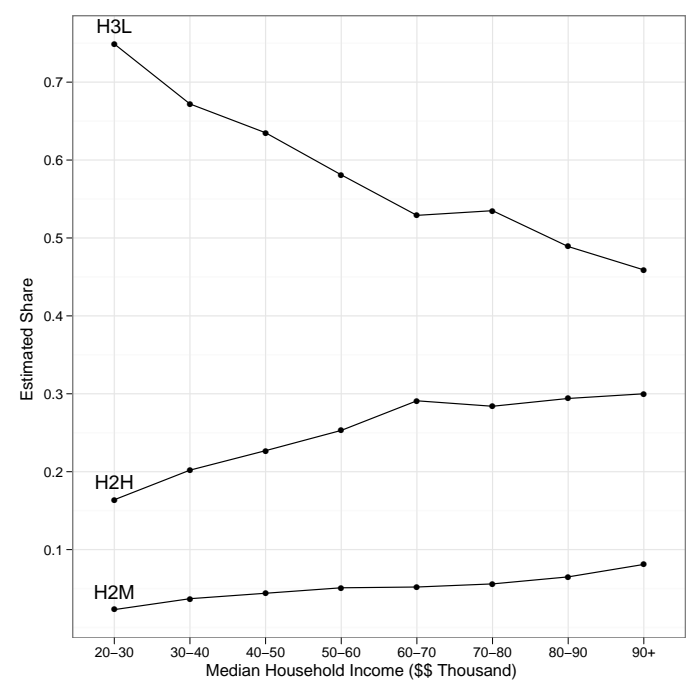


Figure 3: Localization Lift versus Maximum Total SKUs in the Assortment and Chain Optimal Share
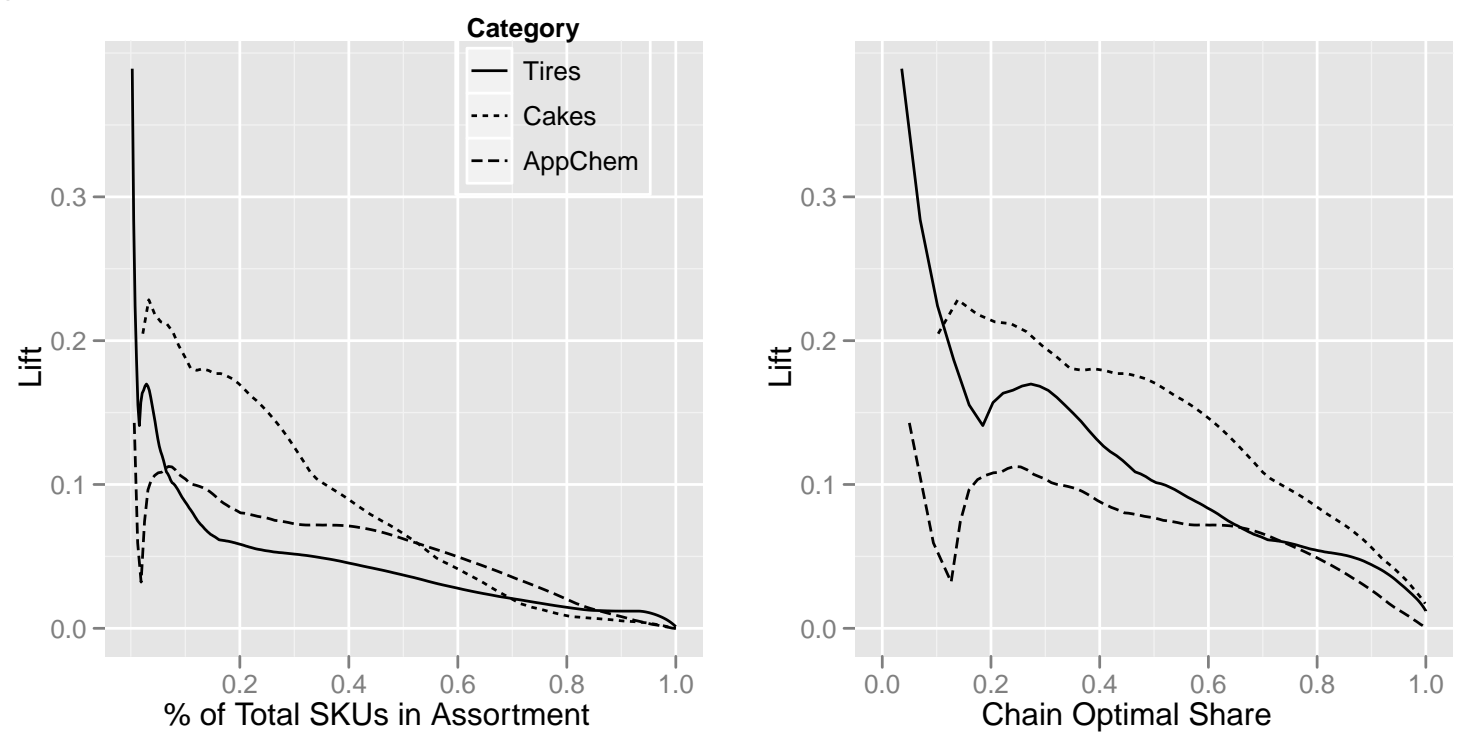

\section{References}

Anupindi, R., M. Dada, S. Gupta. 1998. Estimation of consumer demand with stock-out based substitution: An application to vending machine products. Marketing Science 17(4) 406-423.

Bell, D.R., A. Bonfrer, P.K. Chintagunta. 2005. Recovering SKU-level preferences and response sensitivities from market share models estimated on item aggregates. Journal of Marketing Research 42(2) 169-182.

Belloni, A., R. Freund, M. Selove, D. Simester. 2008. Optimizing Product Line Designs: Efficient Methods and Comparisons. Management Science 54(9) 1544.

Beswick, P., M. Isotta. 2010. Making the right choices: SKU rationalization in retail. Oliver Wyman Working Paper.

Cachon, G., C. Terwiesch, Y. Xu. 2005. Retail assortment planning in the presence of consumer search. Manufacturing $\&$ Service Operations Management 7(4) 330.

Canadian Tire Corporation Limited. 2007. Annual report.

Caro, F., J. Gallien. 2007. Dynamic assortment with demand learning for seasonal consumer goods. Management Science 53(2) 276-276.

Chong, J. K., T. H. Ho, C. S. Tang. 2001. A modeling framework for category assortment planning. Manufacturing ES Service Operations Management 3(3) 191-210.

Dobson, G., S. Kalish. 1988. Positioning and pricing a product line. Marketing Science 7(2) 107-125.

Fader, Peter S., Bruce G. S. Hardie. 1996. Modeling consumer choice among skus. Journal of Marketing Research 33(4) 442-452.

Fisher, M., A. Raman. 2010. The New Science of Retailing: How Analytics are Transforming the Supply Chain and Improving Performance. Harvard Business School Press.

Gaur, V., D. Honhon. 2006. Assortment planning and inventory decisions under a locational choice model. Management Science 52(10) 1528-1543.

Goodman, A. C. 1998. Andrew court and the invention of hedonic price analysis. Journal of Urban Economics 44(2) 291-298. 
Green, P.E., A.M. Krieger. 1985. Models and heuristics for product line selection. Marketing Science 4(1) 1-19.

Green, P.E., A.M. Krieger. 1987a. A consumer-based approach to designing product line extensions. Journal of Product Innovation Management 4(1) 21-32.

Green, P.E., A.M. Krieger. 1987b. A simple heuristic for selecting 'good' products in conjoint analysis. Applications of Management Science 5 131-153.

Green, P.E., A.M. Krieger. 1992. An application of a product positioning model to pharmaceutical products. Marketing Science 11(2) 117-132.

Guadagni, P.M., J.D.C. Little. 1983. A logit model of brand choice calibrated on scanner data. Marketing Science 2(3) 203-238.

Hotelling, H. 1929. Stability in competitition. Economic Journal 39 41-57.

Kamakura, W.A., G.J. Russell. 1989. A probabilistic choice model for market segmentation and elasticity structure. Journal of Marketing Research 26(4) 379-390.

Kohli, R., R. Sukumar. 1990. Heuristics for Product-Line Design Using Conjoint Analysis. Management Science 36(12) 1464-1478.

Kök, G., M. L. Fisher. 2007. Demand Estimation and Assortment Optimization under Substitution: Methodology and Application. Operations Research 55(6) 1001-1021.

Kök, G., M. L. Fisher, R. Vaidyanathan. 2008. Assortment planning: Review of literature and industrial practice. Invited chapter in Retail Supply Chain Management, Eds. N. Agrawal, S.A. Smith. Kluwer Publishers.

Lancaster, K. 1975. Socially Optimal Product Differentiation. The American Economic Review 65(4) 567-585.

Lancaster, K. J. 1966. A new approach to consumer behavior theory. Journal of Political Economy 74 132-157.

Maddah, B., EK Bish. 2007. Joint pricing, assortment, and inventory decisions for a retailer's product line. Naval Research Logistics 54(3) 315.

Mahajan, S., G. van Ryzin. 2001. Stocking retail assortments under dynamic consumer substitution. Operations Research 49(3) 334-351.

McBride, R.D., F.S. Zufryden. 1988. An integer programming approach to the optimal product line selection problem. Marketing Science 7 126-140.

McGregor, Jena. May 15, 2008. At Best Buy, marketing goes micro. BusinessWeek .

O'Connell, Vanessa. April 21, 2008. Reversing field, Macy's goes local. Wall Street Journal .

Pakes, Ariel. 2003. A reconsideration of hedonic price indexes with an application to PC's. The American Economic Review 93(5) 1578-1596.

Rosen, S. 1974. Hedonic prices and implicit markets: Product differentiation in pure competition. The Journal of Political Economy 82(1) 34-55.

Smith, S. A., N. Agrawal. 2000. Management of multi-item retail inventory systems with demand substitution. Operations Research 48(1) 50-64.

van Ryzin, G., S. Mahajan. 1999. On the relationship between inventory costs and variety benefits in retail assortments. Management Science 45(11) 1496-1509.

Vulcano, G., G. van Ryzin, R. Ratliff. 2009. Estimating primary demand for substitutable products from sales transaction data. Working Paper, NYU Stern.

Zimmerman, A. September 7, 2006. To boost sales, Wal-Mart drops one-size-fits-all approach. Wall Sreet Journal Online A1.

Zimmerman, Ann. October 7, 2008. Home Depot learns to go local. Wall Street Journal . 